\title{
Evaluation of home bleaching gel modified by different thickeners on the physical properties of enamel: An in situ study
}

\author{
Bruna Guerra Silva ${ }^{1}$, Thayla Helle Nunes Gouveia ${ }^{1}$, \\ Marcelo de Assumpção Pereira da Silva ${ }^{2,3}$, Gláucia Maria Bovi Ambrosano ${ }^{1}$, \\ Flávio Henrique Baggio Aguiar ${ }^{1}$, Débora Alves Nunes Leite Lima ${ }^{1}$
}

Correspondence: Dr. Débora Alves Nunes Leite Lima Email: dalima@unicamp.br
'Department of Restorative Dentistry, Piracicaba Dental School, University of Campinas, Piracicaba, SP, Brazil, 2Paulista Central University Center, Institute of Physics of Sao Carlos, IFSC/USP, São Carlos, SP, Brazil, ${ }^{3}$ Paulista Central University Center, UNICEP, São Carlos, SP, Brazil

\section{ABSTRACT}

Objective: This in situ study evaluated the influence of $10 \%$ carbamide peroxide $(\mathrm{CP})$ in combination with different thickeners on physical characteristics of enamel. Materials and Methods: Eighty-four bovine blocks of enamel and dentin $(4 \mathrm{~mm} \times 4 \mathrm{~mm} \times 2.5 \mathrm{~mm})$ were divided into seven groups $(n=12)$, fixed in palatal intraoral devices, and submitted to different treatments: $\mathrm{CP}+$ carbopol (FGM), CP + carbopol + glycerin (ultradent), $\mathrm{CP}+$ natrosol (experimental gel), carbopol, carbopol + glycerin, and natrosol, without treatment (control group). The physical properties tested were color $(\Delta E)$, roughness (Ra), and Knoop microhardness (KHN). Statistical Analysis Used: Data were submitted to ANOVA, Tukey's, and Dunnett's tests $(\alpha=0.05)$. Results: all groups presented significant color variation, except carbopol. For microhardness, all bleached groups did not differ from control. Roughness increased in all bleached groups when compared to the control group, but $\mathrm{CP}+$ natrosol presented statistical lower results than $\mathrm{CP}+$ carbopol. Conclusion: Replacement of carbopol by natrosol caused less change on roughness surface, at the same time yielding an effective whitening result.

Key words: Bleaching, carbopol, natrosol

\section{INTRODUCTION}

The mechanism of action of bleaching gels relies on oxygen-free radicals ${ }^{[1]}$ released from the decomposition of hydrogen peroxide. The free radicals responsible for the whitening effect are also accountable for the changes in dental physical properties, such as reduction of enamel microhardness. Because of their highly unstable nature and nonspecific reactivity, they

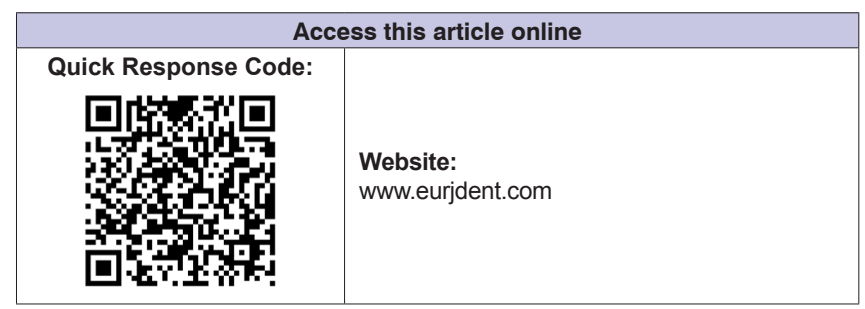

can interact not only with chromogenic molecules, but also with other organic and inorganic molecules of dental tissues, to achieve stability. ${ }^{[2,3]}$ Acidic $\mathrm{pH}$ of bleaching gels can also cause changes in tooth structure. ${ }^{[4]}$ Nevertheless, studies have shown that in addition to free radicals and $\mathrm{pH}$, changes in tooth

This is an open access journal, and articles are distributed under the terms of the Creative Commons Attribution-NonCommercial-ShareAlike 4.0 License, which allows others to remix, tweak, and build upon the work non-commercially, as long as appropriate credit is given and the new creations are licensed under the identical terms.

For reprints contact: reprints@medknow.com

How to cite this article: Silva BG, Nunes Gouveia TH, Pereira da Silva Md, Bovi Ambrosano GM, Baggio Aguiar FH, Leite Lima DA. Evaluation of home bleaching gel modified by different thickeners on the physical properties of enamel: An in situ study. Eur J Dent 2018;12:523-7.

DOI: 10.4103/ejd.ejd_352_17 
structure may also be related to other constituents present in the bleaching product formulation, such as thickeners. ${ }^{[5]}$

Thickeners are chemical substances that have the function of increasing the viscosity of bleaching gels, thus allowing a greater retention on the dental surface and prolonging the exposure time to active compounds. ${ }^{[6]}$ The most commonly used thickeners in the composition of at-home bleaching gels are carbopol and glycerin. However, these thickeners are capable of interacting with the dental structure, leading to structural changes such as reduced microhardness and altered roughness. ${ }^{[2]}$ In this context, the present study evaluates currently marketed thickeners in bleaching gels in comparison to natrosol. Natrosol has the advantages of being nonionic thickener and having a wide-range pH stability (2.0-12.0), ${ }^{[7]}$ which means it can be combined to acidic substances such as bleaching formulations.

This in situ study investigated the effects of an experimental natrosol-containing bleaching gel on the properties and morphology of enamel, evaluating its color, microhardness, and surface roughness. Hypotheses tested were (1) that the experimental bleaching gel with natrosol would show the same efficacy of the commercial bleaching agents with carbopol and glycerin and (2) that the experimental bleaching gel would not change the microhardness and roughness of the enamel.

\section{MATERIALS AND METHODS}

This study was approved by the Institutional Ethics Committee (53165116.1.0000.5418).

\section{Sample preparation}

Enamel/dentin blocks measuring $4 \mathrm{~mm} \times 4 \mathrm{~mm} \times 2.5 \mathrm{~mm}$, with $1 \mathrm{~mm}$ of enamel and $1.5 \mathrm{~mm}$ of dentin, were obtained, using a metallographic cutter (IsoMet 1000, Buehler Ltd, Lake Bluff, IL, USA). For grinding, regularization, and polishing, decanting silicon carbide sanding discs were used (\#1200/2500/p4000-IsoMet, Buehler Ltd, Lake Bluff, IL, USA), and then, felts with metallographic diamond pastes and specific lubricant $(6 \mu \mathrm{m}, 3 \mu \mathrm{m}, 1$ $\mu \mathrm{m}$ Arotec, Cotia, SP, Brazil) were used for polishing. Samples were stained for 6 days by immersion in black tea solution (Lion Junior SA, Curitiba, PR, Brazil), which was changed every $24 \mathrm{~h}$. The specimens were sterilized with ethylene oxide and stored in water at $4^{\circ} \mathrm{C}$ until use.

\section{In situ settings}

Twelve volunteers (6 males and 6 females), aged 24-28 years, participated in the study after signing a free and informed consent form. All of these volunteers were selected by the inclusion criteria (normal salivary flow, measured through sialometry, and the absence of caries and/or periodontal disease), without violating the exclusion criterion (use of medicine that interferes with salivary flow, smoking habits, and the presence of orthodontic device, fixed or removable prosthesis). For each volunteer, a molding in alginate (Hydrogum-Zhermack, Badia Polesine, Italy) of the upper arch was obtained, from which the palatal devices were made of acrylic resin containing seven reservoirs ( $25 \mathrm{~mm}^{2}$ wide and 3-mm deep). Seven specimens (enamel/dentin blocks) were attached to each palatal device using sticky wax. Intraoral devices remained for 1 day in the volunteers' mouths before the beginning of the bleaching sessions, for the formation of acquired film.

\section{Treatment application}

A total of 14 daily treatment sessions were carried out as follows: devices were removed from the volunteers' mouths; six of the seven specimens contained in each of the 12 devices were submitted to gel application, according to the assigned experimental group, whereas the seventh, control specimens did not receive any treatment. Gels were applied according to the manufacturer's recommendations on the enamel surface, remaining for $4 \mathrm{~h}$ at room temperature. After each session, the gel was removed with cotton-tipped flexible rods, specimens were washed with water, and the devices were returned to the volunteers' mouths.

\section{Color analysis}

The color reading of each specimen was performed in ambient light condition (GTI MiniMatcher MM-1, GTI Technology, New York, USA) using a spectrophotometer (CM 700D, Minolta, Osaka, Japan). The spectral distribution was measured based on the CIE system $L^{*}, a^{*}$, and $b^{*}$, used in Color software (Konica Minolta). The difference between initial (baseline) and final ( $24 \mathrm{~h}$ after treatment) $L^{*}$, $a^{*}$, and $b^{*}$ values was obtained $(\Delta L, \Delta a$, and $\Delta b)$, and total color change was calculated using the following equation: $\Delta E=\left(\left[\Delta L^{*}\right] 2+\left[\Delta a^{*}\right] 2+\left[\Delta b^{*}\right] 2\right) 1 / 2$.

\section{Microhardness analysis}

The enamel microhardness was analyzed using a Knoop penetrator with a $50 \mathrm{~g}$ load and a $5 \mathrm{~s}$ time in a microhardness tester (HMV-2000, Shimadzu, 
Tokyo, Japan). Five penetrations were made in each specimen at $100 \mu \mathrm{m}$ each, and the mean was calculated to determine the Knoop hardness number (KHN).

\section{Roughness analysis}

Roughness (Ra) was analyzed using a profilometer (Surfcorder 1700, Kosaka, Japan) at two time-points: initial (baseline) and $24 \mathrm{~h}$ after completion of treatment. Three equidistant directions were measured on the surface of each sample, with a cutoff point of $0.25 \mathrm{~mm}$, a reading length of $1.25 \mathrm{~mm}$, and a velocity of $0.1 \mathrm{~mm} / \mathrm{s}$. Results were expressed as the difference between the two time-points $(\Delta \mathrm{Ra})$.

\section{Atomic force microscopy}

For quantitative analysis of the surface, two samples from each group were randomly chosen and analyzed by atomic force microscopy (AFM) (MultiMode 3; Digital Instruments Bruker, Santa Barbara, USA) after the treatment.

\section{Statistical analyses}

The data were submitted to exploratory analysis, including boxplots. Afterward, $\Delta E$ data were analyzed, using ANOVA (factorial plus control $-2 \times 3+1$ ) and Tukey's test. The comparisons with the control were performed by the Dunnett's test. Because all volunteers received all treatments, the "volunteer effect" was accounted for during these analyses $(\alpha=0.05)$.

\section{RESULTS}

\section{Color $(\Delta E, \Delta L, \Delta a$, and $\Delta b)$}

Table 1 refers to the color change $(\Delta E)$ and Figure 1 refers to all coordinates of the color change $(\Delta L, \Delta a, \Delta E$, and $\Delta b$ ). All groups presented higher $\Delta E$ than control, except for carbopol. Carbamide peroxide (CP) showed higher $\Delta E$ than the respective nonbleached groups, regardless of the thickener used. Natrosol presented higher $\Delta E$ values when compared to carbopol. Carbopol + glycerin gel yielded results which were intermediate, being statistically equivalent to both natrosol and carbopol groups.

\section{Microhardness (KHN)}

The results of microhardness measurements are shown in Table 2. Experimental treatments did not cause significant changes in microhardness compared to control, except for carbopol and carbopol + glycerin in the absence of bleaching, which presented lower microhardness values than the others.

\section{Roughness (Ra)}

The effects of experimental treatments on surface roughness are shown in Table 3. CP differed statistically in roughness from the control. When comparing bleached versus nonbleached, the firsts presented higher roughness, regardless of the thickener. Among the groups treated in the presence of $\mathrm{CP}$, the use of natrosol yielded the lowest values of $\Delta \mathrm{Ra}$ when compared to the carbopol. CP + carbopol + glycerin did not differ from the natrosol and carbopol.

\section{Atomic force microscopy}

The three-dimensional representative images of the enamel surface show morphological changes in all bleached groups compared to the control [Figure 2a-d] measured by AFM.

\section{DISCUSSION}

Data showed that the group treated with PC + natrosol showed greater color variation, and presented greater

\begin{tabular}{|c|c|c|}
\hline \multirow[t]{2}{*}{ Thickener } & \multicolumn{2}{|c|}{$10 \%$ carbamide peroxide } \\
\hline & With & Without \\
\hline Carbopol & *5.87 (1.25) Ab & $2.39(0.79) \mathrm{Bb}$ \\
\hline Carbopol + glycerin & *6.74 (1.71) Aab & *2.95 (1.14) Bab \\
\hline Natrosol & *6.83 (1.24) Aa & *3.28 (1.32) Ba \\
\hline Control & \multicolumn{2}{|c|}{$1.68(0.60)$} \\
\hline
\end{tabular}

Table 2: Mean (standard deviation) of microhardness

\begin{tabular}{lcc}
\hline Thickener & \multicolumn{2}{c}{$\mathbf{1 0 \%}$ carbamide peroxide } \\
\cline { 2 - 3 } & With & Without \\
\hline Carbopol & $310.01(20.92) \mathrm{Aa}$ & ${ }^{*} 249.69(30.25) \mathrm{Bb}$ \\
Carbopol + glycerin & $309.34(13.60) \mathrm{Aa}$ & ${ }^{*} 260.29(37.81) \mathrm{Bb}$ \\
Natrosol & $318.72(27.16) \mathrm{Aa}$ & $307.57(15.26) \mathrm{Aa}$ \\
Control & \multicolumn{2}{c}{$322.28(16.58)$} \\
\hline
\end{tabular}

*It differs from the control group $(P \leq 0.05)$. Means followed by distinct letters (upper case in horizontal and lower case in vertical) differ from each other $(P \leq 0.05)$

\begin{tabular}{lcc} 
Table 3: Mean (standard deviation) of the roughness \\
\cline { 2 - 3 } Thickener & \multicolumn{2}{c}{$\mathbf{1 0 \%}$ carbamide peroxide } \\
\cline { 2 - 3 } & ${ }^{*} 0.043(0.008) \mathrm{Aa}$ & $0.014(0.005) \mathrm{Ba}$ \\
Carbopol & ${ }^{*} 0.042(0.008) \mathrm{Aab}$ & $0.011(0.006) \mathrm{Ba}$ \\
Carbopol + glycerin & ${ }^{*} 0.034(0.009) \mathrm{Ab}$ & $0.011(0.009) \mathrm{Ba}$ \\
Natrosol & \multicolumn{2}{c}{$0.012(0.006)$} \\
Control
\end{tabular}

*It differs from the control group $(P \leq 0.05)$. Means followed by distinct letters (upper case in horizontal and lower case in vertical) differ from each other $(P \leq 0.05)$ 


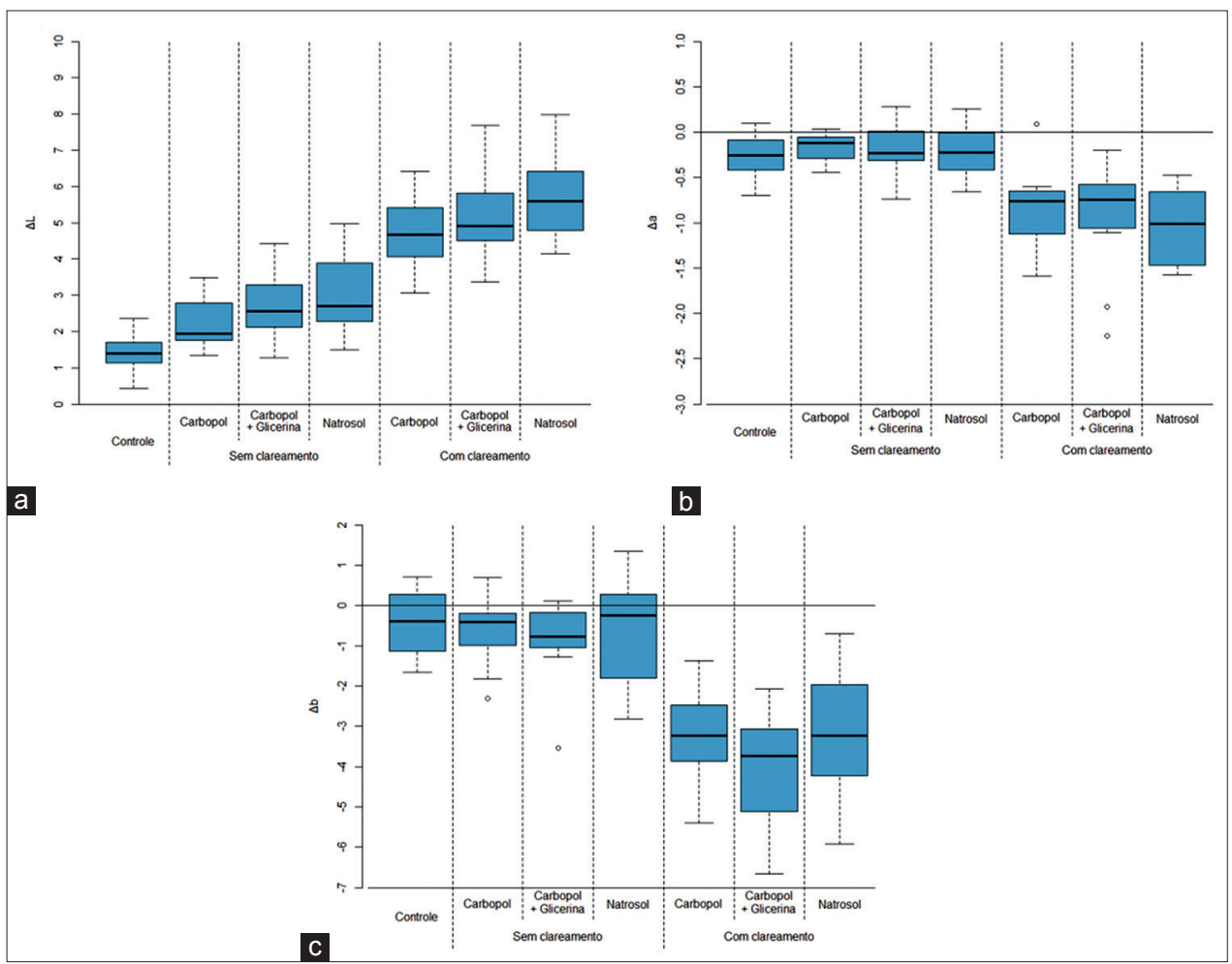

Figure 1: Boxplot of: (a) $\Delta L$; (b) $\Delta a$; and (c) $\Delta b$

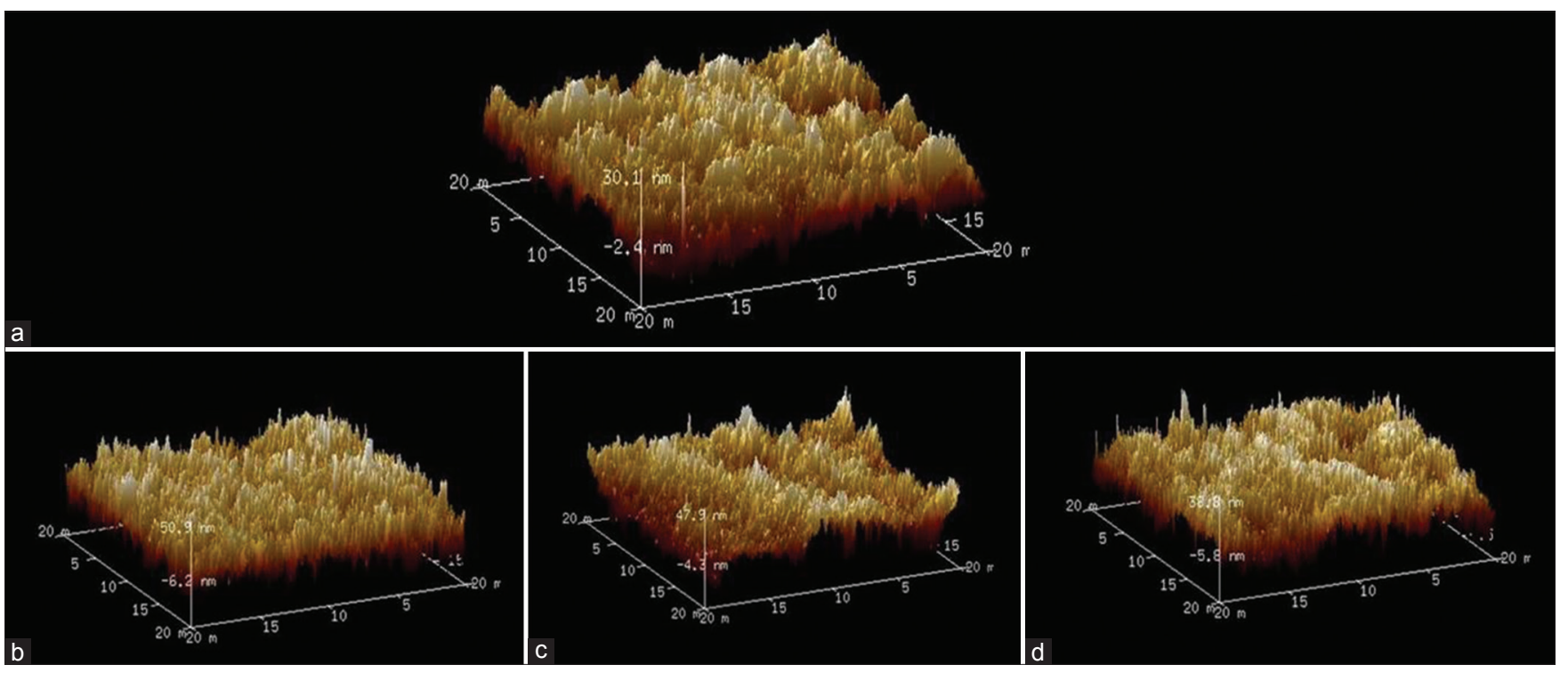

Figure 2: Images obtained by atomic force microscopy. Morphological changes could be observed in all bleached groups compared to the control (a), regardless of the thickener used (b) (carbamide peroxide + carbopol), (c) (carbamide peroxide + carbopol + glycerin), and (d) (carbamide peroxide + natrosol)). However, carbamide peroxide + natrosol (d) presented a less pronounced surface alteration when compared with carbamide peroxide + carbopol (b), as demonstrated by the smaller difference in peak-valley measurements on the enamel surface

$\Delta E$ values when compared to $\mathrm{PC}+$ carbopol. In addition to the bleaching activity of $\mathrm{CP}$ in bleaching gels through the interaction of oxygen-free radicals with chromogenic molecules in the tooth structure, the alteration of the enamel surface, such as increased roughness, can lead to change in the values of $\Delta E$, presenting lower values of $\Delta E$. As the gel with
PC + natrosol presented lower roughness values, different from PC + carbopol gel, the increased color change effect of $\mathrm{PC}+$ natrosol gel can be justified when compared to PC + carbopol.

Natrosol (hidroxietilcelulose) is a cellulose-based polymer and differs from carbopol mainly due to its nonionic 
nature ${ }^{[7]}$ In addition, natrosol can be associated to acidic substances in whitening gels, due to its remarkable stability to $\mathrm{pH}$ variation (2.0-12.0). Carbopol, in turn, a thickener commonly used in at-home bleaching gels, ${ }^{[7]}$ is a carboxypolymethylene polymer, ionic and acidic in nature, being a carboxylic acid derivative. Due to its strong calcium-binding ability, ${ }^{[8]}$ it has been reported that carbopol can reduce enamel microhardness and dentin since it causes inhibition of hydroxyapatite crystal incorporation. In addition to carbopol, other thickener also already used in whitening gels is glycerin, which because of its ability to act as a barrier for adsorption of saliva and consequent remineralization of enamel, can also affect the microhardness of enamel surfaces. ${ }^{[2]}$

Previous studies have reported a decrease in enamel microhardness after whitening treatment with $\mathrm{CP},[9,10]$ often associated to demineralization effect of free radicals resulting from dissociation of $\mathrm{CP}^{[6]}$ and. ${ }^{[11]}$ In the present study, only the groups treated with carbopol and carbopol + glycerin in the absence of $\mathrm{CP}$ presented a decreased microhardness. This suggests that interaction of oxygen-free radicals with enamel may not be the only cause for decreased microhardness in dental structures. ${ }^{[5,12]}$ Interestingly, natrosol used either alone or in association with $\mathrm{CP}$ did not cause changes in microhardness of enamel, since no statistically significant differences were found in comparison to the control group.

Regarding roughness, changes in physical properties can occur due to effects of demineralization and remineralization. Demineralization can be caused by diffusion of hydrogen peroxide after dissociation of $\mathrm{CP}$ and by acidic $\mathrm{pH}$ of whitening products ${ }^{[13]}$ and thickeners. Remineralization of treated surface takes place when bleached enamel is in contact with human saliva, because of deposition of minerals such as calcium and phosphate. ${ }^{[14]}$ However, this process takes place in an irregular way, inducing reorganization of the enamel prisms that can cause an increase of roughness, as observed in the present study. Given the different $\mathrm{pH}$ values of the different thickeners, more acidic compounds may be responsible for greater demineralization effect. Consequently, during remineralization, there would be more extensive repositioning of enamel prisms due to mineral deposition on demineralized surface. This is in accordance to this study findings - groups treated with carbopol, a relatively acidic compound, presented higher roughness values when compared to natrosol.

\section{CONCLUSION}

The use of carbopol, glycerin, and natrosol did not cause a significant alteration on enamel microhardness during bleaching, yielding an effective whitening result. However, the replacement of carbopol by natrosol caused less change on roughness surface.

\section{Acknowledgment}

The collaboration between Drogal manipulation, FGM dentistry products, and ACECIL sterilization. The authors declare no potential conflicts of interest with respect to the authorship and/or publication of this article.

\section{Financial support and sponsorship \\ Nil.}

\section{Conflicts of interest}

There are no conflicts of interest.

\section{REFERENCES}

1. Eimar H, Siciliano R, Abdallah MN, Nader SA, Amin WM, Martinez PP, et al. Hydrogen peroxide whitens teeth by oxidizing the organic structure. J Dent 2012;40 Suppl 2:e25-33.

2. Basting RT, Rodrigues AL Jr., Serra MC. The effect of $10 \%$ carbamide peroxide, carbopol and/or glycerin on enamel and dentin microhardness. Oper Dent 2005;30:608-16.

3. Kawamoto K, Tsujimoto Y. Effects of the hydroxyl radical and hydrogen peroxide on tooth bleaching. J Endod 2004;30:45-50.

4. Sun L, Liang S, Sa Y, Wang Z, Ma X, Jiang T, et al. Surface alteration of human tooth enamel subjected to acidic and neutral $30 \%$ hydrogen peroxide. J Dent 2011;39:686-92.

5. Basting RT, Rodrigues AL Jr., Serra MC. The effects of seven carbamide peroxide bleaching agents on enamel microhardness over time. J Am Dent Assoc 2003;134:1335-42.

6. McCracken MS, Haywood VB. Demineralization effects of 10 percent carbamide peroxide. J Dent 1996;24:395-8.

7. Gouveia TH, Públio JC, Ambrosano GM, Paulillo LA, Aguiar FH, Lima DA, et al. Evaluation of physical properties of a nanocomposite after aging, bleaching and staining. J Appl Biomater Funct Mater 2016;14:e256-65.

8. van der Reijden WA, Buijs MJ, Damen JJ, Veerman EC, ten Cate JM, Nieuw Amerongen AV, et al. Influence of polymers for use in saliva substitutes on de- and remineralization of enamel in vitro. Caries Res 1997;31:216-23.

9. Potocnik I, Kosec L, Gaspersic D. Effect of $10 \%$ carbamide peroxide bleaching gel on enamel microhardness, microstructure, and mineral content. J Endod 2000;26:203-6.

10. Shannon H, Spencer P, Gross K, Tira D. Characterization of enamel exposed to $10 \%$ carbamide peroxide bleaching agents. Quintessence Int 1993;24:39-44.

11. Sa Y, Chen D, Liu Y, Wen W, Xu M, Jiang T, et al. Effects of two in-office bleaching agents with different $\mathrm{pH}$ values on enamel surface structure and color: An in situ vs. in vitro study. J Dent 2012;40 Suppl 1:e26-34.

12. Rodrigues JA, Marchi GM, Ambrosano GM, Heymann HO, Pimenta LA. Microhardness evaluation of in situ vital bleaching on human dental enamel using a novel study design. Dent Mater 2005;21:1059-67.

13. Tezel H, Ertaş OS, Ozata F, Dalgar H, Korkut ZO. Effect of bleaching agents on calcium loss from the enamel surface. Quintessence Int 2007;38:339-47.

14. Humphrey SP, Williamson RT. A review of saliva: Normal composition, flow, and function. J Prosthet Dent 2001;85:162-9. 